\title{
Arabidopsis thaliana outer ovule integument morphogenesis: Ectopic expression of KNATI reveals a compensation mechanism Elisabeth Truernit ${ }^{* 1,2}$ and Jim Haseloff ${ }^{1}$
}

\author{
Address: ${ }^{1}$ University of Cambridge, Department of Plant Sciences, Downing Site, Cambridge CB2 3EA, UK and ${ }^{2}$ INRA, Centre de Versailles, Institut \\ Jean-Pierre Bourgin, Laboratoire de Biologie Cellulaire, Route de St-Cyr, 78026 Versailles cedex, France \\ Email: Elisabeth Truernit* - etruernit@versailles.inra.fr; Jim Haseloff - jh295@cam.ac.uk \\ * Corresponding author \\ Published: 14 April 2008 \\ BMC Plant Biology 2008, 8:35 doi:10.1 I86/147|-2229-8-35 \\ This article is available from: http://www.biomedcentral.com/I47I-2229/8/35 \\ (C) 2008 Truernit and Haseloff; licensee BioMed Central Ltd. \\ This is an Open Access article distributed under the terms of the Creative Commons Attribution License (http://creativecommons.org/licenses/by/2.0), \\ which permits unrestricted use, distribution, and reproduction in any medium, provided the original work is properly cited. \\ Received: 21 January 2008 \\ Accepted: 14 April 2008
}

\begin{abstract}
Background: The Arabidopsis outer ovule integument is a simple two-cell layered structure that grows around the developing embryo and develops into the outer layer of the seed coat. As one of the functions of the seed coat is the protection of the plant embryo, the outer ovule integument is an example for a plant organ whose morphogenesis has to be precisely regulated.

Results: To better characterise outer ovule integument morphogenesis, we have isolated some marker lines that show GFP expression in this organ. We have used those lines to identify distinct cell types in the outer integument and to demonstrate similarities between leaves and the outer integument. Using confocal microscopy, we showed that cell sizes and shapes differ between the two cell layers of the outer integument. Expression of KNATI in the integuments leads to extra cell divisions specifically in the outer layer of the outer integument. This is being compensated for by a decrease of cell volume in this layer, thus showing that mechanisms exist to control proper ovule integument morphogenesis.

Conclusion: The Arabidopsis outer ovule integument can be used as a good model system to study the basic principles of plant organ morphogenesis. This work provides new insights into its development and opens new possibilities for the identification of factors involved in the regulation of cell division and elongation during plant organ growth.
\end{abstract}

\section{Background}

Fertilised ovules develop into seeds that contain the plant embryo. In Arabidopsis thaliana, three distinct regions can be identified along the proximal-distal axis of the ovule primordium (Figure 1). The most proximal structure of the primordium is the funiculus, which connects the primordium to the placenta. At the distal end of the primordium lies the nucellus in which the megaspore mother cell develops. The chalaza in the central zone of the primordium initiates two integuments, each composed of two cell layers $[1,2]$. During ovule development, the two integ- uments grow around the nucellus and, after fertilization, develop into the seed coat that encloses the embryo (Figure 1). Whereas the inner integument initially develops as a radially symmetrical structure that surrounds the nucellus, the outer integument grows only from the side of the ovule primordium that faces the basal end of the carpel (gynobasal side) [1,2]. The outer integument remains two-cell layered throughout seed development $[1,2]$. At later stages of seed development, cells of the abaxial (outer) layer of the outer integument differentiate termi- 


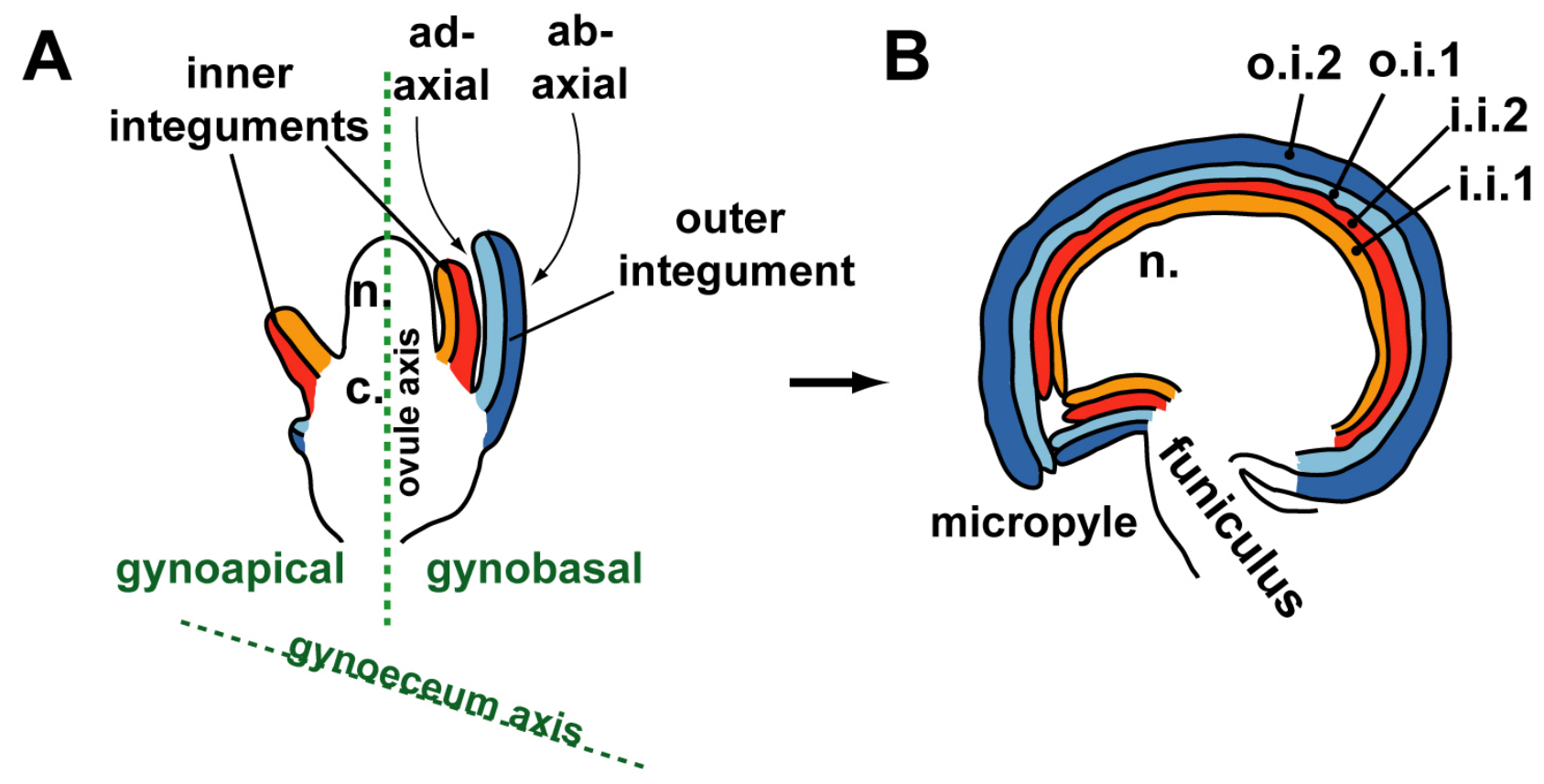

\section{Figure I}

The development of ovule integuments in Arabidopsis. (A) Two inner and one outer integument grow out from the chalaza (c) during early ovule development. (B) Ovule at stage of fertilization: Integuments have grown around nucellus (n), i.i. I: inner (adaxial) layer of inner integument, i.i.2: outer (abaxial) layer of inner integument, o.i. I: inner (adaxial) layer of outer integument, o.i.2: outer (abaxial) layer of outer integument.

nally into highly specialized seed coat cells that contain polysaccharide mucilage $[3,4]$.

The integuments are the only lateral organs produced by the ovule. The evolutionary origin of the integuments is still a matter of debate. The telome theory suggests that integuments originated from the fusion of sterile or fertile branches (telomes) $[5,6]$. It is generally believed that the inner and outer integument derived independently. While the inner integument most likely originated directly from the fusion of telomes or sporangiophores, the outer integument is believed to have developed later from a cupule, a leaf-like structure surrounding one or more ovules $[7,8]$.

The development of the Arabidopsis outer ovule integument involves the same basic processes required for the formation of other determinate lateral plant organs, such as leaves. The outer ovule integument is an example for an organ of determinate growth and characteristic form in which the rate and direction of cell division and elongation needs to be precisely regulated. Asymmetric growth and differentiation are also essential features of its development. In case of the integuments, proper morphogenesis is especially critical, as an improper curvature or closure would lead to seeds with embryos that are not sufficiently protected. However, it seems that integument extension is relatively sensitive to alterations in cell division or cell expansion. Mutations in SHORT INTEGUMENTS2 (SIN2), for example, lead to shorter integuments due to a reduction of cell number [9]. The result of mutations in SIN1/DCL1, on the other hand, show reduced integument size due to a lack of cell expansion $[1,10,11]$.

Because of its simple two-layered structure, the outer integument is an ideal organ for the study of the basic principles of plant morphogenesis. For this a better characterisation of outer integument growth and cell fates within the integument is required. To address this, we have identified Arabidopsis enhancer-trap lines with specific expression of the gene for green fluorescent protein (GFP) in distinct domains of the outer integument. These lines provided good markers for the characterisation of cell proliferation and differentiation during development of the outer integument. KNAT1 is a homeodomain protein that is normally expressed in the shoot apical meristem (SAM), and which alters leaf morphology when ectopically expressed in leaves $[12,13]$. Misexpression of KNAT1 caused increased cell division specifically in the abaxial layer of the outer integument and showed that compensatory mechanisms exist in the outer integument to ensure its proper morphogenesis. 


\section{Results \\ A screen for marker lines for the study of outer integument development}

To obtain markers for the study of outer ovule integument development, a population of 400 Arabidopsis C24 enhancer-trap lines [14] was screened for GFP expression in the outer ovule integument. Six lines showed stable patterns of GFP expression in this tissue. In seed coats of seeds that contained walking stick stage embryos, GFP expression in three of the lines (KS059, KS110, KS151) was seen throughout the outer layer of the outer integument, one line (KS149) showed expression in both outer integument cell layers, and two lines (M214, M237) showed GFP expression that was restricted to the micropylar end of the outer layer of the outer integument. Four of the lines (KS110, KS151, KS149, M237) were chosen for a more detailed analysis of GFP expression patterns throughout ovule and seed development using confocal laser-scanning microscopy (ovules and seeds of three independent plants, $\mathrm{n} \geq 5$ /stage).
To describe GFP expression patterns in those four lines we will follow a recent suggestion by Skinner et al. [15]. We will use the term "gynobasal" to refer to the side of the ovule primordium that faces the base (receptacle) of the carpel, and the term "gynoapical" for the side that faces the apical (stigma) side of the carpel. The terms "abaxial" and "adaxial" will be used to refer to the polarity of the lateral organs of the ovule, i.e. the integuments (see Figure $1)$.

\section{Markers for the adaxial-abaxial polarity in the outer integument}

GFP expression in ovules of line KS110 was restricted to the abaxial (outer) layer of the outer integument (o.i.2). Expression started before integument outgrowth in the epidermis of the funiculus (Figure 2A). As the outer integument grew out, GFP was first only expressed at the chalazal end (Figure 2B). During early embryogenesis, GFP expression extended throughout the o.i.2. Expression persisted in this layer during the later stages of seed coat development (Figure $2 \mathrm{C}$ to $2 \mathrm{~F}$ ). GFP was also found in the L1 layer of the nucellus during early stages of ovule devel-
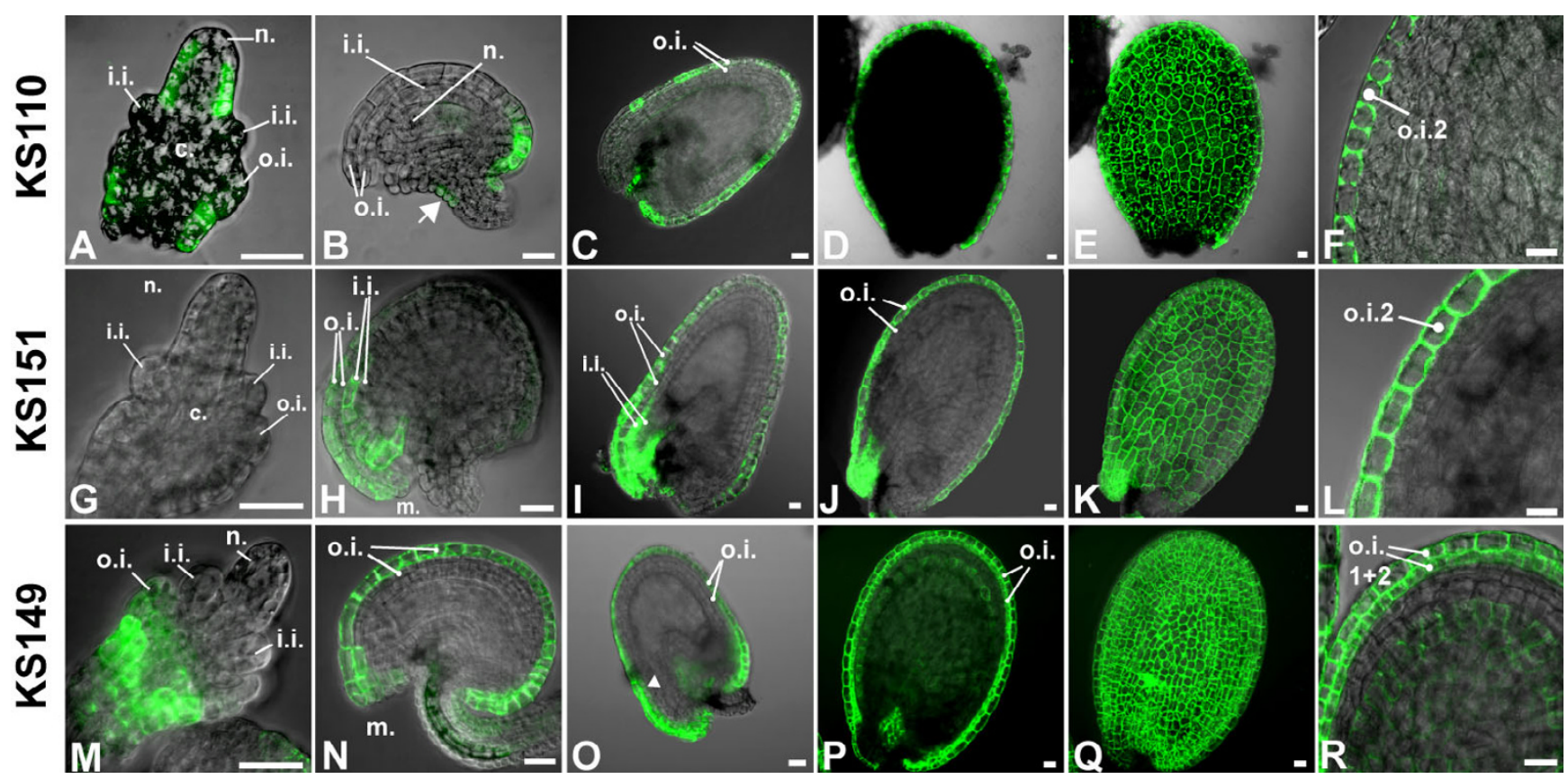

Figure 2

Confocal laser-scanning images of GFP expression patterns during ovule and seed development in enhancertrap lines KS I I 0, KS I 5 I, and KS I49. (A) to (F) GFP expression in line KSI IO. (A), (B) Ovule development: GFP is expressed in the abaxial layer of the outer ovule integument and in a subset of cells on the gynoapical side of the funiculus region (arrow). (C) to (F) Seed development: GFP is expressed throughout the o.i.2. (G) to (L) GFP expression in line KSI5I. (G) No GFP expression is seen during early ovule development. (H) After fertilization, GFP can be seen at the micropylar end of both integuments in the abaxial cell layers. (I) to (L) Late seed development: GFP is expressed on the micropylar end of the i.i.2 and throughout the o.i.2. (M) to (R) GFP expression in line KSI49. (M), (N) GFP is initially expressed only in the o.i.2. (O) to (R) During seed development, GFP expression is also seen in the o.i. I. The arrow in (O) shows beginning of expression in the o.i. I. (E), (K), and (Q) are overlay projection images of (D), (J), and (P), respectively. (F), (L), and (R) show details of outer integument expression Scalebars: $20 \mu \mathrm{m}$. 
opment (Figure 2A). The expression in the abaxial layer of the outer integument resembled the expression of INO $[16,17]$. Unlike INO expression, however, the KS110 marker was also expressed in a small subset of cells in the epidermal layer on the gynoapical side (arrow in Figure 2B).

Line KS151 also showed GFP expression in the o.i.2. Expression started around the time of fertilization. In contrast to line KS110, GFP was initially expressed only at the micropylar end (Figure 2H). Later it could be seen throughout the outer layer of the outer integument (Figure 2I to 2L). In addition, line KS151 also exhibited GFP expression in the abaxial cell layer of the inner integuments, where expression remained restricted to the micropylar end throughout seed development.

In contrast to lines KS110 and KS151, line KS149 showed expression of GFP in both outer integument layers. GFP fluorescence was observed before integument outgrowth in the region immediately underneath the chalaza (Figure $2 \mathrm{M})$. In the early stages of ovule development, GFP expression was only seen in the o.i.2 (Figure 2N). During early seed development GFP fluorescence then was also detected in the o.i.1. Expression of GFP remained in both outer integument layers during the late stages of seed coat development. Faint GFP expression was also seen in the endothelium cell layer (Figure 2P, R).

\section{The markers also label adaxial-abaxial cell layers in shoot tissues}

Lines KS110, KS151, and KS149 showed expression of GFP in other lateral organs with the same axial preference as in the outer integument. In leaves and petals of KS110 plants expression of GFP was restricted to the abaxial epidermis (Figure 3C to 3E). Line KS151 showed GFP expression mainly in the leaf petiole. Again, expression was only found in the abaxial epidermis (Figure $3 \mathrm{~F}$ to $3 \mathrm{H}$ ) with erratic individual cells expressing GFP on the adaxial side. In KS149 leaves and petals, GFP was expressed strongly in the epidermis. Like in the outer ovule integument, it did not show any axial preferences (Figure 3I to $3 \mathrm{~K}$ ).

\section{A marker for the distal region of the abaxial outer integument cell layer}

Cells at the distal portion of the outer integument (the micropylar end) are visibly more elongated and are therefore distinct from the cells of the rest of the integument. GFP fluorescence in line M0237 was first detected around fertilization and was restricted to these cells throughout seed development (Figure 4). GFP expression in line M0237 therefore specifically marked this cell type. The M0237 marker was not expressed in leaves or petals.

\section{Cells in the adaxial and abaxial cell layer of the outer integument differ in size and shape}

We took advantage of confocal microscopy, which makes it possible to image individual cell layers without the need for physical tissue sectioning. Line KS149, which shows GFP expression in both outer integument cell layers, was used to visualize cells in the o.i.1 and o.i.2. Seeds with globular stage ( 4 to 8 cell stage) embryos were analysed. Images of GFP expressing cells in the o.i.1 and o.i.2 were taken separately (Figure 5A, B). In 3 seed coats analysed, cell areas of the $0 . i .2$ were significantly $(\mathrm{p} \leq 0.0001)$ larger than those of the o.i.1 [see Additional file 1]. In addition, the majority of cells in the outer layer were 7-sided, while the inner layer had more 6-sided cells [see Additional file $1]$.

\section{Ectopic expression of KNAT I causes extra cell divisions and reveals a compensatory mechanism during outer ovule integument morphogenesis}

Over-expression of KNOX homeodomain proteins confers indeterminancy on normally determinate organs, such as leaves $[12,13,18]$. Ectopic expression of KNAT2 in ovules led to the homeotic conversion of the nucellus into carpeloid structures in a Landsberg erecta (Ler) background (Pautot et al. 2001). To investigate ovule development in KNAT1 over-expressing plants, the KNAT1 cDNA was translationally fused to the gene of the yellow fluorescent protein YFP and put under the control of the constitutive CAMV 35S promoter [19]. To ensure nuclear localization of KNAT1, a nuclear localisation sequence (NLS) derived from the SV40 T-antigen [20] was added to the KNAT1YFP fusion. Twenty independent Arabidopsis lines (ecotype C24) were obtained. Eleven of the lines showed the characteristic lobed leaf phenotype that had been described previously for KNAT1 over-expressing plants $[12,13]$. Three lines with strong leaf lobing were chosen for further analysis of the T3 and T4 generation (lines 13, 41, and 51). Nuclear localized KNAT1-YFP fluorescence could be seen in all cells of the ovules of these lines throughout all development stages (not shown).

To analyse seed morphology, seeds were stained with the fluorescent dye safranin $\mathrm{O}$ and viewed with the confocal microscope. Seeds of the KNAT1 over-expressing plants showed two obvious morphological differences to wild type C24 seed:

1) The shape of an Arabidopsis wild type seed resembles an ellipsoid with the poles being at and opposite the side where the funiculus was attached. In wild type seed, the integuments closed up with the funiculus approximately in the middle of the funicular side of the seed. In seeds of KNAT1 over-expressers this closing was shifted towards the gynobasal side (see arrows in Figure 6A, B). 


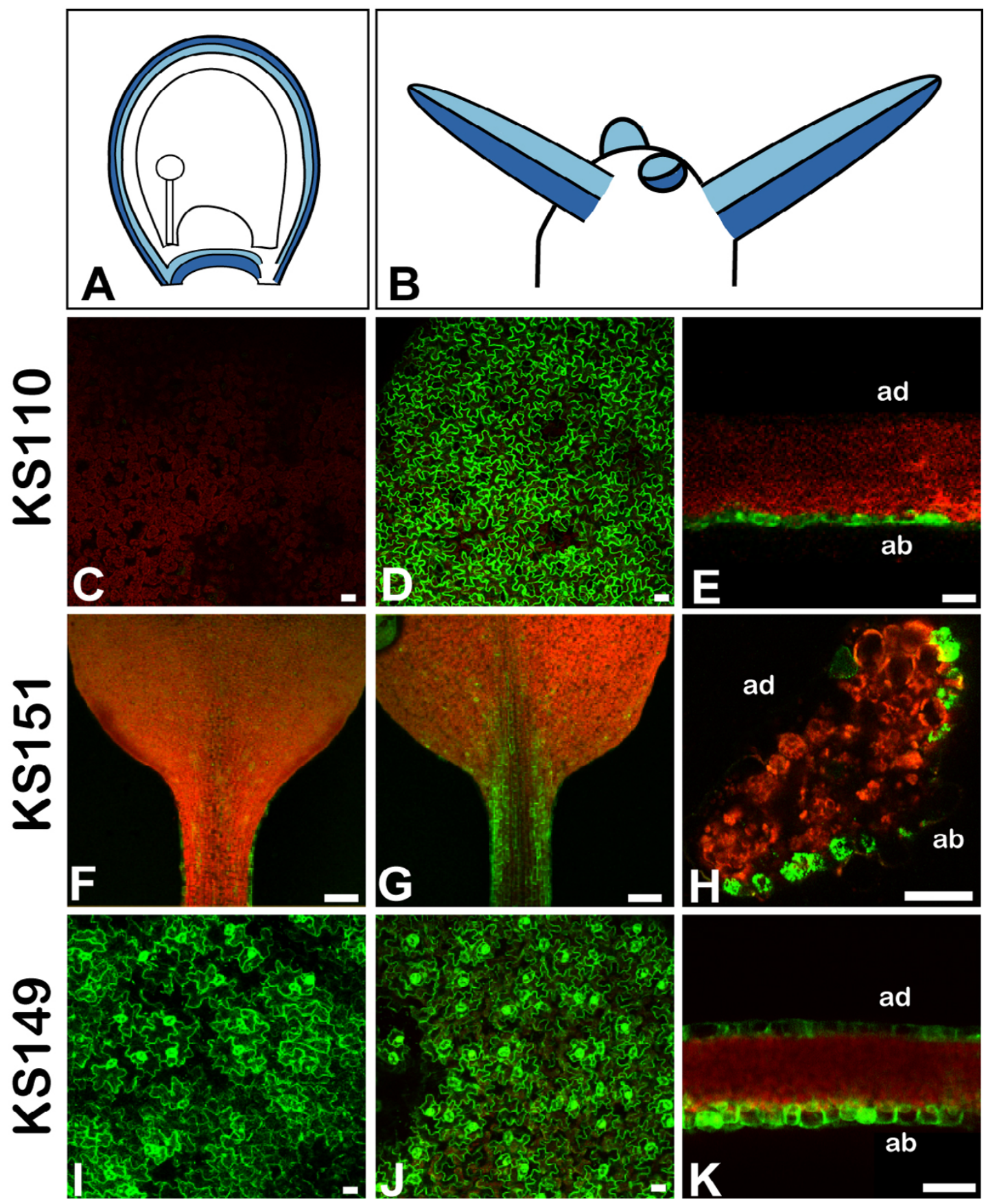

Figure 3

Confocal laser-scanning images of GFP expression patterns in leaves of enhancer-trap lines KS I I 0, KS I 5 I, and KSI49 show similarities to ovule expression. (A) Abaxial (dark blue) and adaxial (light blue) domains of the outer integument. (B) Abaxial and adaxial domains of lateral organs of the shoot apical meristem (colour code as in (A)). (C) to (E) GFP is only expressed in the abaxial domain of lateral organs in line KSI IO. (C) No GFP expression is seen in the adaxial epidermis of KSI I 0 leaves (red colour is chlorophyll auto-fluorescence). (D), (E) Strong GFP expression is seen in the abaxial leaf epidermis. (F) to (H) GFP is only expressed in the abaxial domain of lateral organs in line KSI5I. (F) Adaxial epidermis of leaf petiole showing no GFP expression. (G), (H) Abaxial epidermis of petiole with GFP expression. (I) to (K) GFP expression in line KSI49 marks abaxial and adaxial domains of lateral organs. Adaxial (I) and abaxial (J) leaf epidermis shows GFP expression. (C), (D), (F), (G), (I), and (J) show surface views, while (E), (H), and (K) show sections through (E), (K) the leaf lamina or (H) the petiole of the marker lines. Scalebars: $20 \mu \mathrm{m}$, in (F) and (G): $100 \mu \mathrm{m}$. 

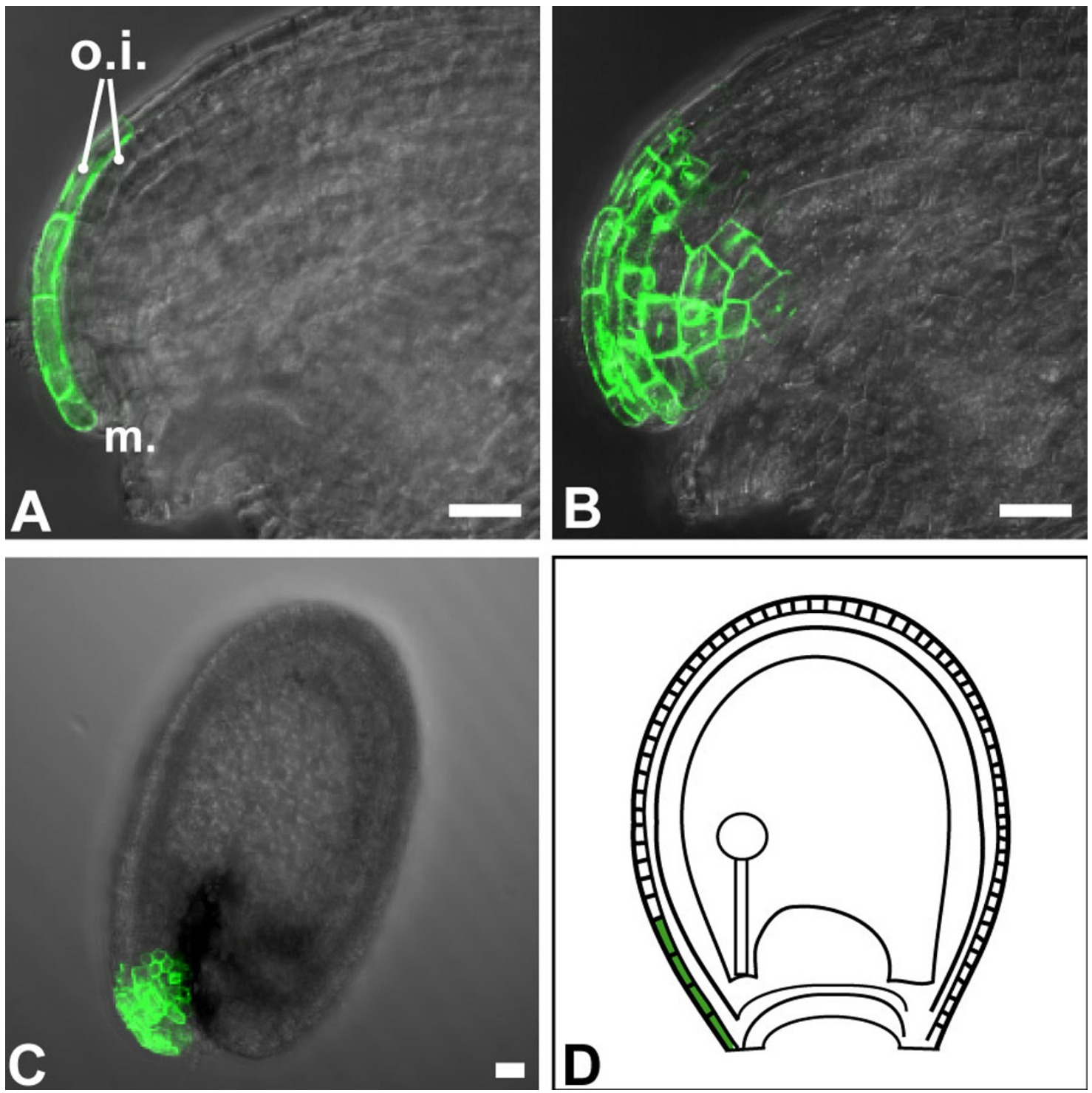

\section{Figure 4}

GFP expression in line M0237 during seed development. GFP expression marks the long cells of the o.i.2 at the micropylar end of the outer integument. (B) Overlay projection of (A). (C) Overlay projection of mature seed showing persistence of marker gene expression. (D) Cells with distinct identity are located at the distal end of the ovule and seed integuments.

Scalebars: $20 \mu \mathrm{m}$.

2) Seed coat cell sizes were obviously reduced in KNAT1 over-expressing lines (Figure 6A, B). Seed coat cell area sizes $(n \geq 25)$ of three seeds of three KNAT1 over-expressing lines were measured and compared to wild type. Cell areas in the seed coat of the KNAT1 over-expressing lines were about half the size of the wild type cell areas (Figure $6 \mathrm{C})$. This difference was highly significant (p-values: 35SK1-13: 0.0025, 35SK1-41: 0.0052, 35SK1-51: 0.0036). Since seed sizes of wild type and KNAT1 misexpressing lines were not different (not shown), ectopic expression of
KNAT1 thus caused the formation of about twice as many cells in the outer seed coat layer.

Reduced cell size was not a general feature of KNAT1 overexpression. Cell areas were measured in the abaxial and adaxial layers of the epidermis of mature petals ( $n$ of cells $\geq 22$ per petal, 6 petals of 3 plants were analysed). No difference in petal cell area sizes could be detected between KNAT1 over-expressers and wild type (not shown). 

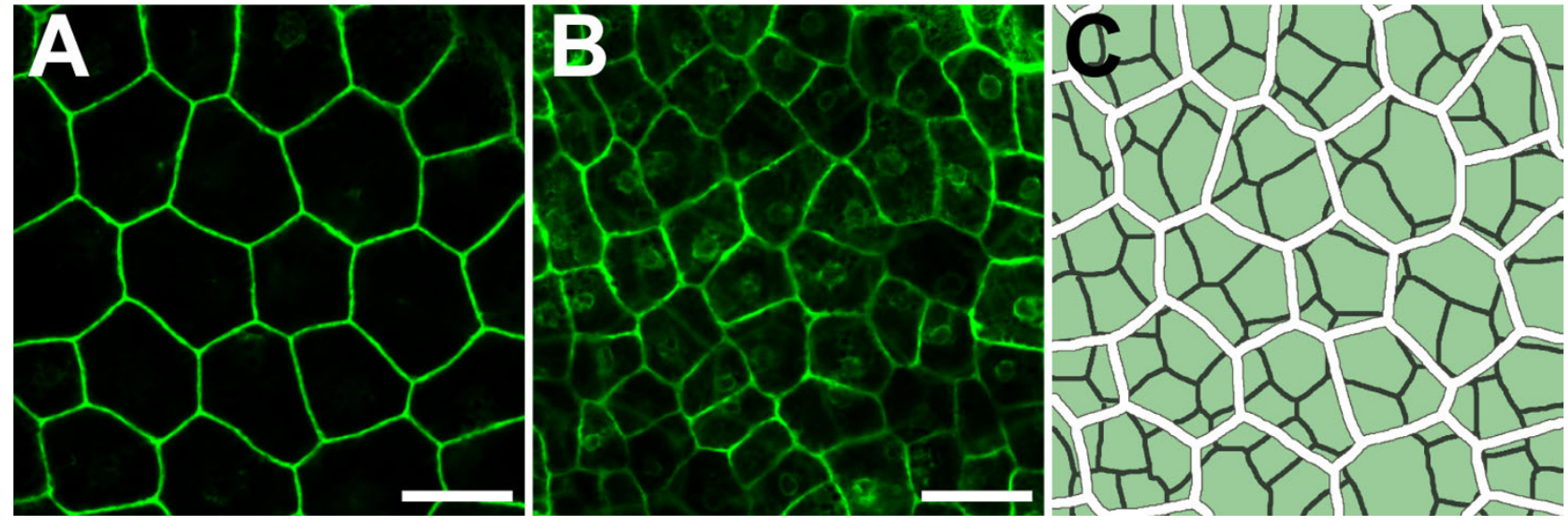

Figure 5

Confocal laser-scanning images of GFP expression in the outer integument cell layers of line KS I 49. GFP expression in the o.i.2 (A) and the o.i.I (B) of line KSI49. The embryo was at globular stage in this seed. (C) Overlay image of (A) and (B), cell walls of the o.i.2 are coloured in white, cell walls of the o.i. I in dark grey. Scalebars: $20 \mu \mathrm{m}$.

To compare the KNAT1 overexpression phenotype with the phenotype reported for overexpression of KNAT2, we also introduced the 35S:KNAT1-YFP-NLS construct into a Ler background. Reduced seed coat cell sizes similar to those observed in the $\mathrm{C} 24$ background were detected (Figure 6C), but no homeotic conversions were observed.

The seed coat was normal in plants transgenic for a 35S:KNAT5-YFP-NLS construct (C24 background) (Figure $6 C ;[21])$. Thus, we can exclude the formal possibility that YFP in the nucleus interferes with normal cell proliferation.

\section{Extra cell divisions in KNAT I over-expressing plants occur specifically in the abaxial layer of the outer integument after fertilisation}

To follow seed coat development in the KNAT1 overexpressing plants, we crossed the enhancer trap lines M0237, KS110, KS149, and KS151, and a line that constitutively expresses a plasma membrane localised form of GFP $[22,23]$ into the KNAT1 over-expressing lines. For comparison, the marker lines were also backcrossed into C24. Developing ovules and seeds were analysed in the F1 generation.

The progeny of crosses of the membrane-marker line to the KNAT1 over-expressing plants were used to analyze cell area sizes of the abaxial layer (o.i.2) before fertilization. Cells in the outer layer of the outer integument had the same size in wild type and KNAT1 over-expressing plants (Figure 7A to 7E).

Crosses to line KS149 were used to analyse cell sizes of both outer integument layers after ovule fertilization.
Images of both cell layers of seeds of different developmental stages were taken. Cell area measurements showed that only the cells in the abaxial layer of the outer integument of KNAT1 over-expressing plants were smaller than in wild type. From early embryo development onwards, the ratio of abaxial:adaxial cell area sizes was $2.4(+/-$ $0.328): 1$ in a wild type background and $1.27(+/-0.194): 1$ in a KNAT1 over-expressing background (Figure 7F to 7J) (p-values for 3 arbitrary chosen data points: $\leq 0.0001$ ). These numbers suggest that, on average, cells underwent one extra cell division in the o.i. 2 of the KNAT1 overexpressing plants around or shortly after fertilization. Therefore, in KNAT1 over-expressing lines, the size of cells in the abaxial layer of the outer integument was more similar to those of the adaxial cell layer.

We can exclude that the different response to KNAT1 misexpression in the two cell layers of the outer integument was the result of different activities of the 35S promoter used for KNAT1 misexpression in those layers: The 35S promoter showed uniform expression throughout ovule development [see Additional file 2].

\section{Ectopic expression of KNATI causes altered marker gene expression}

Crosses of line KS110 to the KNAT1 over-expressing lines resulted in plants with markedly reduced expression of GFP in the o.i.2. GFP was only expressed in a subset of random cells, and the outcome of the expression was later in relation to expression of GFP in a KS110 line that was backcrossed to C24 (Figure 8E, F). In contrast, the KNAT1YFP-NLS fusion was normally expressed throughout the o.i.2, excluding cosuppression of the fluorescent protein genes (not shown). This shows an alteration of cell fate in 

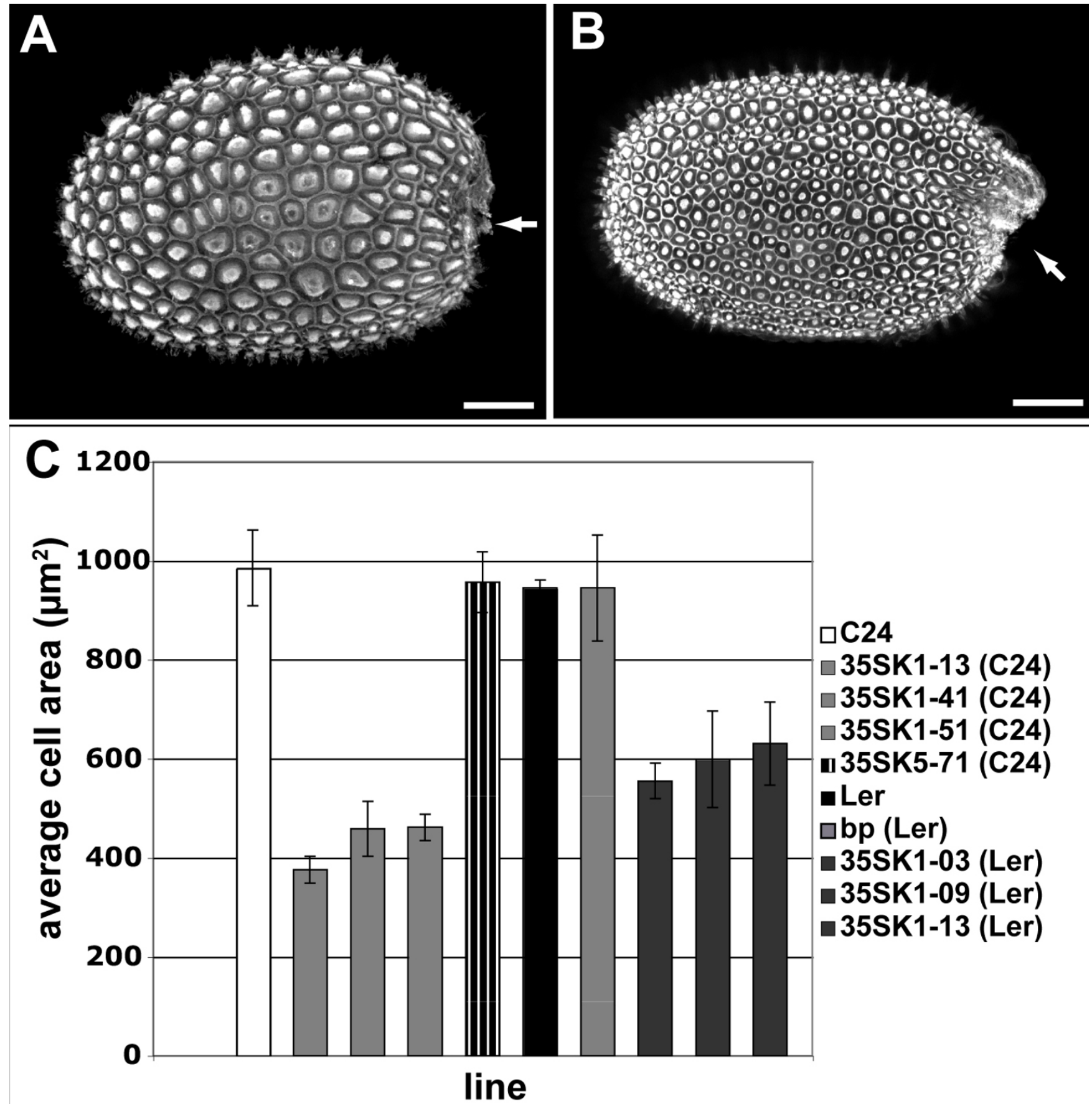

Figure 6

Seed coat phenotype of KNATI misexpressing plants. Confocal images of seed coats stained with safranin O. (A) Wild type seed coat. (B) Example of seed coats of a KNATI over-expressing line. Images are overlay projections. Arrows show the positioning of the integument closure. Scalebars: $100 \mu \mathrm{m}$. (C) Average area of cells in the outer seed coat of mature Arabidopsis seed of different transgenic lines and different ecotypes. Shown are data for ecotypes C24 and Landsberg erecta (Ler), for different KNATI overexpression lines (35SKI-xx) in C24 and Ler backgrounds, for a KNAT5 overexpression line (35SK5-7I) in C24 background, and for brevipedicellus (bp) in Ler background. For each data point $25-30$ cells of 3 different seeds were measured. Error bars show standard error of the means. 

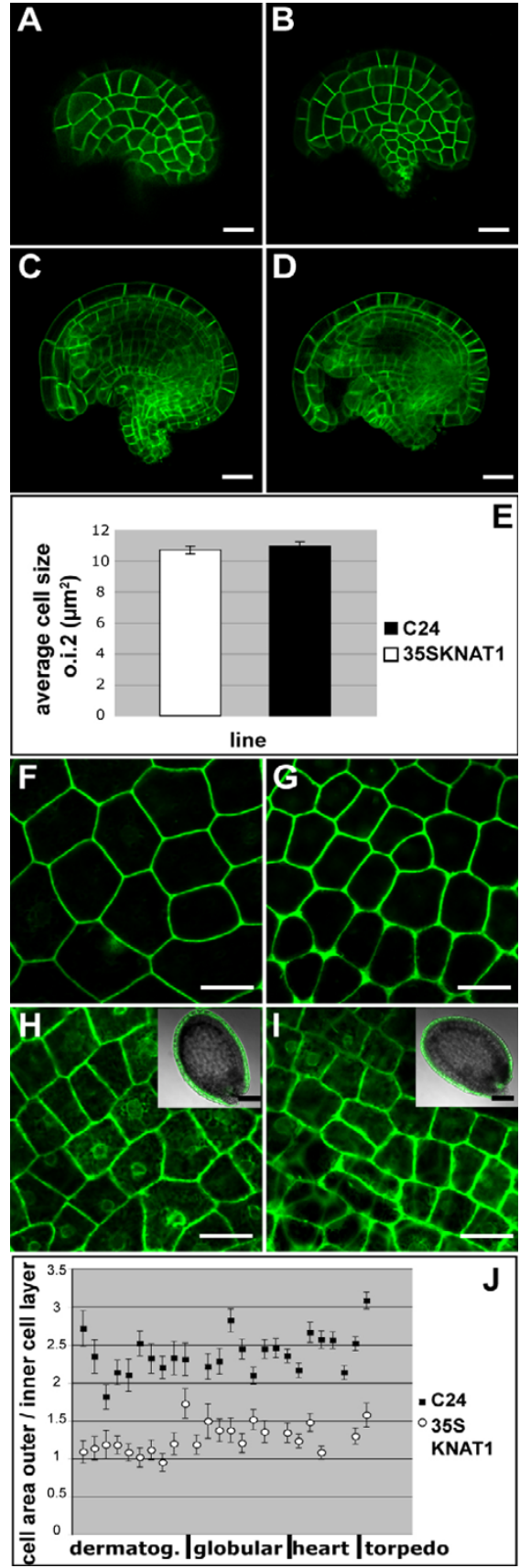

\section{Figure 7}

Outer integument cell areas in wild type and KNATI misexpressing lines at different stages of development. (A) to (E) Seed coat cell sizes of ovules at the stage of fertilization expressing a fusion between GFP and a membrane-localized protein. (A) Outer integument of wild type ovule. (B) Outer integument of ovule of KNATI misexpressing plant. (C) and (D) are optical sections of (A) and (B), respectively. (E) Cell area measurements of o.i. 2 cells shows no difference in cell area between wild type (black) and KNATI misexpressing (white) plants at this stage. (F) to (J) Seed coat cell area sizes of ovules after fertilization. (F) to (I) Seeds with glob ular stage embryos. (F) o.i.2 and (H) o.i.I of line KSI49. (G) o.i.2 and (I) o.i.I of ular stage embryos. (F) o.i.2 and (H) o.i. I of line KSI49. (G) o.i.2 and (I) o.i.I of
KNATI over-expressing plants crossed to line KSI 49 . Cells in the o.i.2 of KNATI misexpressing plants are visibly smaller. (J) Ratio of cell area sizes in the o.i.2 versus the o.i. I of wild type and KNATI misexpressing lines at different stages of seed deve opment. Shown are measurements of integument cell areas $(n=25-30)$ of individual developing seeds related to the developmental stage of the embryo. Black squares: wild type. White circles: KNATI misexpressing lines. Scalebars: $20 \mu \mathrm{m}$, in inserts 100 $\mu \mathrm{m}$. the abaxial cell layer. Down-regulation of the KS110 marker was specific for the integument, as it was not observed in leaves of KNAT1 over-expressing plants.

\section{Over-proliferation of cells in the outer integument results in a more pronounced hyponastic growth of the integument}

During wild type seed development, the micropylar end and the chalazal end of the integuments are approximately levelled. This is reflected by the shape of the endothelium. Figure 8A, B shows seeds of wild type and KNAT1 over-expressers during early embryo development. The endothelium accumulates proanthocyanidins and can therefore be selectively stained with vanillin. Unlike in wild type, the micropylar end was positioned below the chalazal end in the ovules of plants overexpressing KNAT1.

The same was seen in line M0237 that had been crossed to KNAT1 over-expressing plants. With ectopic expression of KNAT1, the region of GFP expression in M0237 at the micropylar end of the integument was shifted towards the gynobasal side. In addition, the GFP expression domain was longer than in wild type because more cells expressed GFP at the micropylar end (Figure 8C, D). Unlike the rest of the integument, cells at the micropylar end did not seem to be able to compensate fully for the extra cell division by reducing their size. Therefore more cells of almost normal size were expressing the GFP marker.

In summary, although reduced cell elongation largely compensated for the extra cell division in the o.i.2 of plants with ectopic expression of KNAT1, a more pronounced hyponastic growth of the integument was still noticeable. The shape of the seed of KNAT1 over-expressing plants was slightly distorted, with the closure of the integuments shifted towards the gynobasal side (see Figure $6 \mathrm{~A}, \mathrm{~B}$ ). As a result of the ovule bending, more tissue was exposed on the funicular end that was not protected by a seed coat (see Figure 9A, B).

In mature Arabidopsis seeds the embryo lies bent with the root tip at the micropylar end and the cotyledon tips at the chalazal end of the seed [24]. Therefore the root tip lies closest to the unprotected area of the KNAT1 over-expressing plant seed. We noticed that seedlings of KNAT1 overexpressing lines were often impaired in root growth and developed secondary roots at a much earlier stage (Figure 9G). These seedlings seemed to have localized tissue damage in the embryonic root tip and occasionally also at the tip of the cotyledons (Figure 9C to 9F). To quantify our observations, seeds $(\mathrm{n}=30)$ of C24 and KNAT1 overexpressing lines were germinated and the number of seedlings with impaired root growth was counted. While wild type roots grew normally, 3.5\% (line 41 ) and $7.1 \%$ (line 

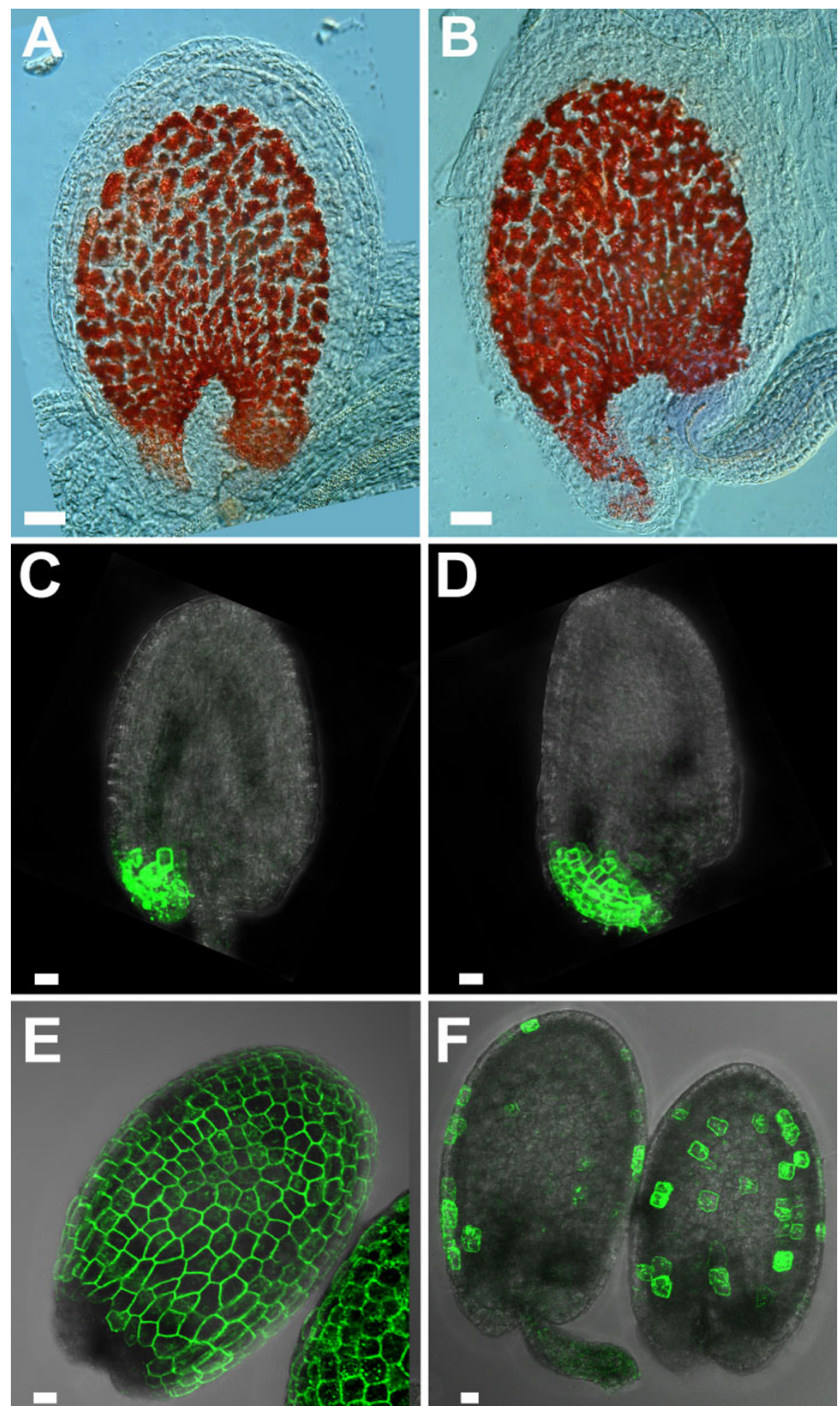

Figure 8

Outer integument shape and cell fate changes in KNATI misexpressers. (A), (C), (E) Wild type. (B), (D), (F) KNATI over-expressers. (A), (B) Vanillin staining of the endothelium shows the altered shape of ovules of KNATI misexpressing plants. (C), (D) GFP expression in crosses to line M0237. The domain of GFP expression in KNATI over-expressing plants is enlarged. (E), (F) GFP expression in crosses to line KSIIO. Marker expression is repressed in plants misexpressing KNATI. Scalebars: $10 \mu \mathrm{m}$ 

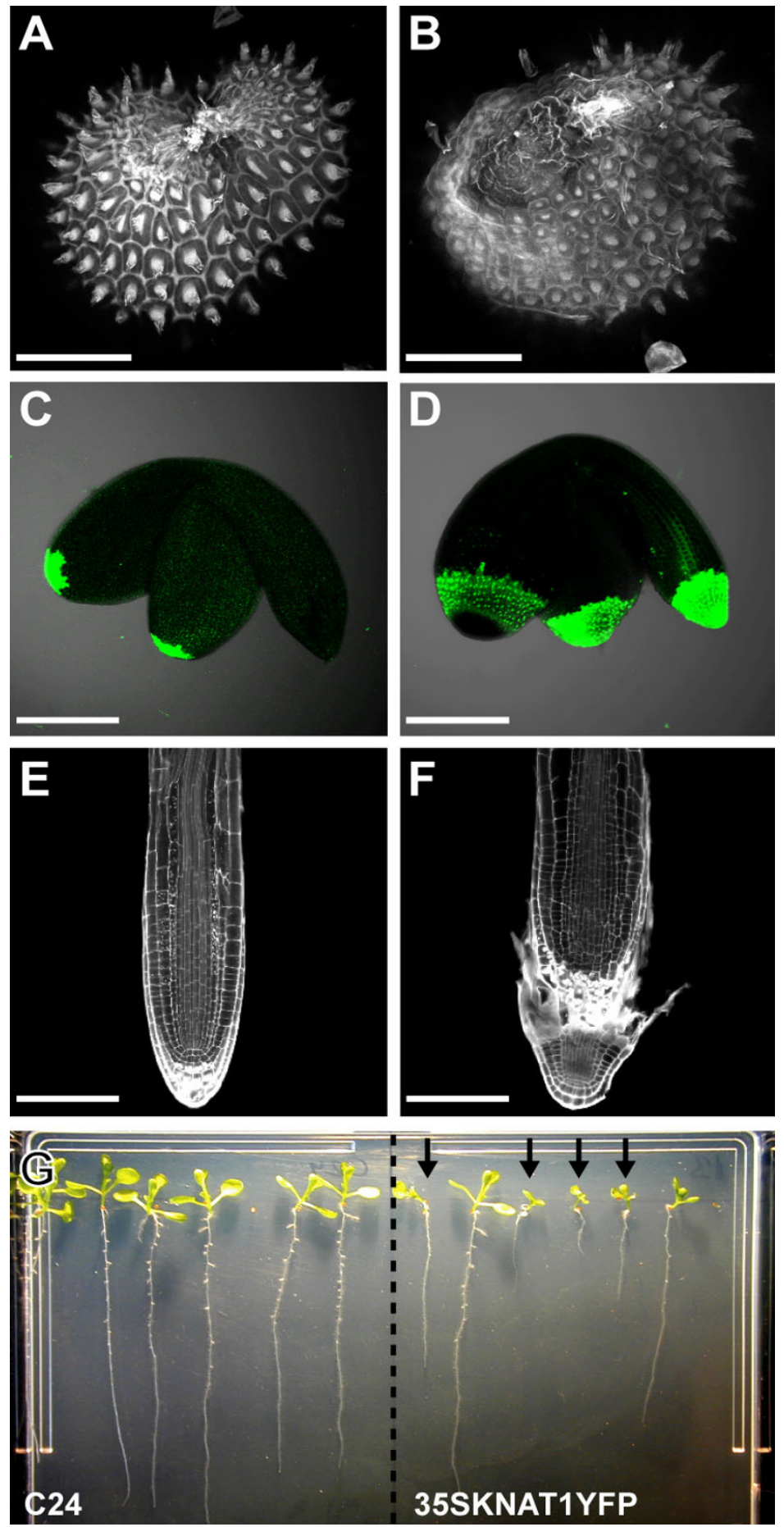

\section{Figure 9}

Consequences of KNATI misexpression for seedling growth. (A), (C), (E) Wild type. (B), (D), (F) KNATI overexpressing plants. (A), (B) Closure of integuments in seeds stained with safranin $O$. The integuments are not fully closed in plants that misexpress KNATI. (C), (D) Sytox green staining of dead cells in embryos. Dead cells can be seen in the embryonic root tip of KNATI over-expressers. (E), (F) 3 day old roots stained with propidium iodide. Development of the embryonic root tip in KNATI over-expressers has not progressed. (G) Wild type and KNATI over-expressing plants grown on vertical plates for 10 days. Seeds were Na-hypochlorite treated for $10 \mathrm{~min}$. Root growth of some KNATI over-expressing seedlings is impaired (arrows). Scalebars: $100 \mu \mathrm{m}$. 
13) of seedlings of the KNAT1 over-expressers showed impaired growth. Sodium hypochlorite treatment, as commonly used for the surface sterilization of seeds, dramatically increased the number of seedlings with severely impaired root growth. Treatment with Na-hypochlorite for 5 minutes led to $20 \%$ and $36 \%$ seedlings with damaged root tips, respectively (wild type control: $3.5 \%$ ). An exposure for 30 minutes resulted in more than $90 \%$ of seedlings with non-viable root tips in both transgenic lines (wild type control: 6.6\%). Therefore, the seed coats of KNAT1 over-expressing plants provided less effective protection against the bleach solution, and cells at the site of closure of the integuments were most vulnerable to damage.

\section{Discussion \\ Domains of gene expression in the outer integument}

We have selected four Arabidopsis enhancer-trap lines [14] for the study of gene expression domains in the outer ovule integument. These lines expressed GFP under the control of endogenous enhancers, depending on the insertion of the enhancer-trap construct into the plant genome. The lines obtained in this study can be used for the analysis of ovule development, as has been shown here for the analysis of KNAT1 misexpressing ovules.

Two main points can be made from the study of GFP expression in the enhancer-trap lines: 1) The different cell identities of cells in the o.i. 1 and in the o.i. 2 are laid down during early integument development and are maintained. Lines KS110 and KS149 showed GFP expression in the o.i. 2 but not in the o.i.1 of the developing outer integument in ovules before fertilization. INO also shows a similar expression pattern $[16,17]$. The establishment of polarity in the integuments might be important for the outgrowth of the integuments, as has been shown for leaves (for a recent review see [25]). In contrast to INO, GFP expression in lines KS149 and KS110 did not show axial preferences with respect to the gynoecium's axis. Lines KS110 and KS151 showed GFP expression in the outer layer of the outer integument also throughout seed development. The differences between o.i.1 and o.i.2 become obvious during late seed coat development when only cells of the o.i.2 differentiate into mucilage containing seed coat cells $[3,4]$.

2) Cells at the micropylar end of both seed integuments are distinct from the rest of the integument cells. These cells also show obvious morphological differences, as they are noticeably longer than the average integument cells [1]. Moreover, they responded differently to KNAT1 misexpression. The morphological differences were reflected by the expression of markers that were not present in the rest of the integument. Line M0237 specifically marked cells at the micropylar end of the o.i.2 after fertilization and during seed development. Line KS151 marked this area in the outer cell layer of both integuments. It is likely that cells at the micropylar end have specific functions such as the protection of the embryo, which is growing from the micropylar end. Moreover, the region includes cells that are among the earliest to undergo elongation during integument growth. The differential expansion of these cells may help bend this tissue to form the characteristic shape of the ovule.

\section{Similarities of leaf and outer integument polarity}

The expression patterns of our marker lines support the theory that the outer integument developed from a leaflike structure $[7,8]$. While lines KS110, KS149, and KS151 with GFP expression in the outer integument did not show expression in the inner integuments, they all showed GFP expression in leaves and some also in other leaf-like structures, such as petals. Moreover, the markers that were expressed on the abaxial side of the outer integument (KS110 and KS151) also showed the same polar expression in leaves. So far, no gene has been found that is exclusively expressed in the o.i.1. To date, we also have not identified an enhancer-trap line that shows GFP expression solely in the o.i.1 (unpublished results). Surprisingly, it has been shown recently that PHABULOSA $(P H B)$, a homeodomain gene that is expressed on the adaxial side of leaves [26,27], is expressed in the inner integument [28]. This led to the speculation that the two integuments of the bitegmic Arabidopsis ovule might have been derived through the splitting of one integument of a unitegmic precursor [28]. According to this, however, we would expect GFP expression in line KS149 to be found in both integuments, as GFP was expressed in all leaf cell layers in this line. In order to clarify these contradicting findings we need to investigate the expression patterns of more ovule-expressed genes on a whole plant level.

\section{KNATI overexpression phenotype}

Many genes that play a role in SAM development are also important for ovule development and vice versa. Although KNAT1 is not normally expressed in ovules $[13,29,30]$, it is likely that KNAT1, when expressed in the ovule, can interact with other proteins that are normal KNAT1 interaction partners in the SAM. Moreover, other KNOX genes could be expressed in the ovule and be involved in the regulation of meristematic activity during integument development. In many cases members of a gene family have become functionally diversified by changes in their expression patterns but their activities are still interchangeable. Several members of the YABBY family, for example, can restore integument outgrowth in ino-1 mutants. However, only the ovule expressed INO is sensitive to SUPERMAN (SUP) regulation that limits INO expression to the gynobasal side of the ovule primordium $[17,31]$. Accordingly, KNAT1 could partially adopt the 
role of another KNOX homeodomain protein but would be insensitive to proper regulatory mechanisms.

In the Arabidopsis SAM, KNAT1, together with STM1, plays a role in the transition zone where it allows for the amplification of stem cell daughter cells by maintaining their meristematic identity before they are consumed by organ formation [32]. In agreement with its role in the SAM, KNAT1 over-expression phenotypes in leaves have been interpreted as being the result of a shift from determinate to indeterminate growth characteristics $[12,13,33]$. In addition, the KNAT1 loss-of-function mutant brevipedicellus $(b p)$ displays defects in cell division in internodes and pedicels, demonstrating that KNAT1 is also required for the maintenance of an indeterminate state in these organs $[29,30]$. Cell differentiation, elongation and growth in the pedicels of $b p$ mutants were more severely affected on the abaxial side than on the adaxial side, causing a change in pedicel growth angle.

Expression of KNAT1 in the ovule integuments triggered ectopic cell divisions, consistent with a role of KNAT1 in maintaining indeterminancy. At present we cannot explain why the presence of KNAT1 only has a visible effect in the o.i.2. However, the phenotype of $b p$ pedicels also seems to suggest that the abaxial side of pedicels is more affected by the presence of KNAT1 than the adaxial side. It is also possible that expression of KNAT1 in the integuments caused a certain degree of adaxialization of the outer integument and therefore the o.i. 2 would adopt o.i.1 features. Two observations support this interpretation. First, expression of KNAT1 in the integuments caused cells of the o.i.2 to divide approximately as often as cells of the o.i.1. Second, a marker that is normally expressed in the o.i.2 but not in the o.i.1 was virtually absent in KNAT1 expressing ovules. As no markers are available that are only expressed in the o.i. 1 we were unable to test the reverse situation.

\section{Regulation of cell proliferation during morphogenesis of the outer integument}

We found clear differences in cell sizes in the two cell layers of the outer integument. Cell area sizes in the o.i.2 were 2.4 times bigger than in the o.i.1, which indicates that during integument development cells in the adaxial layer undergo approximately one division more than cells in the abaxial layer.

Misexpression of KNAT1 almost abolished the differences of cell area sizes in the two layers. Formally, the higher number of smaller cells found in the o.i.2 of integuments of KNAT1 over-expressing plants could be the result of impaired cell elongation or increased cell division. It is more likely that KNAT1 promoted cell division, since cells of the o.i. 2 were still able to elongate after the extra cell division had already occurred. A compensatory mechanism was in place in the o.i.2 that resulted in the restriction of cell expansion and therefore in the development of an outer integument of relatively normal size and shape.

During leaf morphogenesis, mechanisms exist to compensate for defects in cell division or expansion (for review see [34,35]). As a general rule it can be stated that a decrease in cell number or an increase in cell elongation can be compensated for by increased cell expansion or decreased cell division, respectively. So far, there are only a few examples that an increased number of cell divisions or decreased cell expansion, as seen here for KNAT1 expression in the o.i.2, could lead to similar compensatory mechanisms $[35,36]$. To our knowledge a cell layer specific increase of cell divisions, which would more accurately correspond to the KNAT1 overexpression phenotype, has not been achieved so far. Therefore, the KNAT1 overexpression phenotype of ovules represents the first description of a compensatory mechanism that involves a decrease in cell elongation to compensate for increased cell divisions in one specific cell layer. Strict control of integument size on the whole organ level and cell-cell communication between the o.i.1 and the o.i.2 has to be postulated.

For Arabidopsis it has been shown that endosperm growth has a reciprocal effect on integument cell elongation to control final Arabidopsis seed size [37]. Here we show that, in addition to that, growth in the two outer integument cell layers is precisely and independently regulated to achieve coordinated integument morphogenesis. The outer integument would therefore be a good system to study the mechanisms of coordinated cell proliferation that result in the final shape of a plant organ.

\section{Conclusion}

This work shows that previously not recognized compensatory mechanisms exist to ensure proper ovule integument morphogenesis. Moreover, we demonstrate that the outer ovule integument can be used as a good model system to study the basic principles of plant organ morphogenesis. The outer integument is a simple two-cell layered structure, consists of only a few different cell types, and is easily accessible by confocal microscopy. A marker line expressing GFP in both layers of the outer integument enabled us to measure cell areas in the two outer integument cell layers of developing Arabidopsis wild type seeds and can be used for a high-throughput screen for mutants with altered cell division patterns in the integument. Misexpression of KNAT1 in the ovule produces a clear and easily visible phenotype that can be used to identify factors involved in the regulation of cell division and elongation on the organ level. 


\section{Methods}

\section{Transgenic lines}

The GAL4-GFP enhancer trap lines [14] KS110, KS149, KS151, and M0237 are available from the Nottingham Arabidopsis Stock Centre (NASC) [38] as stock numbers N9260, N9266, N9267, and N9339, respectively. Other transgenic lines obtained from the NASC: $b p-1$ (NW30).

\section{Construction of transgenic plants}

The generation of the KNAT1 over-expression construct has been described [21]. Plasmids were electroporated into Agrobacterium tumefaciens GV3101 [39]. Arabidopsis thaliana ecotype C24 and Ler was transformed by floral dip [40]. Transgenic plants were selected on media containing $50 \mathrm{mg} / \mathrm{l}$ kanamycin.

\section{Growth conditions}

Plants were germinated and grown under a $16 \mathrm{~h}$ light, $8 \mathrm{~h}$ dark photoperiod on media containing $0.5 \times$ Murashige and Skoog salt mixture (MS), $0.5 \mathrm{~g} / 1$ 2-(N-morpholino) ethanesulfonic acid (MES) $\mathrm{pH} 5.7$ and $0.7 \%$ agar. For analysis of ovule development plants were grown on soil under constant conditions in the greenhouse.

\section{Confocal laser-scanning microscopy}

Confocal laser-scanning microscopy was performed using a Leica TCS NT/SP microscope. Excitation wavelengths were $488 \mathrm{~nm}$ for GFP, and $514 \mathrm{~nm}$ for YFP.

\section{Seed coat staining}

For staining of mature seed coats with safranin $O$, seeds were incubated in a 1:105 dilution of safranin O (Molecular Probes, Eugene, USA) for $15 \mathrm{~min}$ at room temperature. Seeds were imaged with the confocal laser-scanning microscope with an excitation wavelength of $488 \mathrm{~nm}$ and a collection window of $540-600 \mathrm{~nm}$. Endothelium staining with vanillin was performed according to Nesi et al. [41].

\section{Cell area measurements}

To measure seed coat cell areas, optical sections were taken with the confocal laser-scanning microscope through the outer ovule integument. Cell area measurements were performed on a Macintosh computer using the public domain NIH Image programme (developed at the U.S. National Institutes of Health and available on the Internet [42]).

To measure petal cell areas, petals were cleared with chloral hydrate and images were taken at the microscope with a digital camera. Petal cell areas were measured as described above.

\section{Sodium-hypochlorite treatment}

For seed sterilization and as a functional test of the seed coat, seeds were imbibed in a solution of $2.4 \%$ active sodium hypochlorite (for incubation times see results section). Seeds were washed 2 times with sterile water and plated on growth media.

\section{Authors' contributions}

ET designed, coordinated and carried out the experiments and drafted the manuscript. JH participated in the coordination of the experiments and in drafting the manuscript. All authors read and approved the final manuscript.

\section{Additional material}

\section{Additional file 1}

Size and shape of cells in the outer ovule integument. (A) Cell area measurements of cells in the o.i.2 and o.i.1 of seeds with globular stage embryos show highly significant differences in cell sizes between the layers. (B) Distribution of cell shapes in the two outer integument cell layers. Click here for file

[http://www.biomedcentral.com/content/supplementary/14712229-8-35-S1.jpeg]

\section{Additional file 2}

Activity of $35 S$ promoter in the ovule is uniform. Ovule development visualised in a line that expresses GFP in the plasma membrane under control of the constitutive 35 S promoter. The 35 S promoter shows uniform expression throughout the developmental stages. Scalebars: $20 \mu \mathrm{m}$.

Click here for file

[http://www.biomedcentral.com/content/supplementary/14712229-8-35-S2.jpeg]

\section{Acknowledgements}

We thank M. Bauch and K. Siemering for their assistance in generating the M0237, KSI IO, KSI 49 and KSI5I marker lines. We are grateful to $A$. Navid, J. Stolz, W. Dewitte, J-C. Palauqui, J. Murray, and V. Pautot for helpful suggestions. The work was supported by the Gatsby Charitable Foundation and the BBSRC.

\section{References}

I. Robinson-Beers K, Pruitt RE, Gasser CS: Ovule Development in Wild-Type Arabidopsis and Two Female-Sterile Mutants. Plant Cell 1992, 4(10): I237-1249.

2. Schneitz K, Hülskamp M, Pruitt RE: Wild-type ovule development in Arabidopsis thaliana: a light microscope study of cleared whole-mount tissue. The Plant Journal 1995, 7(5):731-749.

3. Western TL, Skinner DJ, Haughn GW: Differentiation of mucilage secretory cells of the Arabidopsis seed coat. Plant Physiol 2000, I 22(2):345-356.

4. Windsor JB, Symonds VV, Mendenhall J, Lloyd AM: Arabidopsis seed coat development: morphological differentiation of the outer integument. Plant J 2000, 22(6):483-493.

5. Herr JM: The Origin of the Ovule. American Journal of Botany 1995, 82(4):547-564.

6. Zimmermann W: Main results of the "Telome Theory". The Palaeobotanist 1952, I:456-470.

7. Stebbins GL: Flowering plants: evolution above the species level. Cambridge, Massachusetts, Belknap Press of Harvard University; 1974. 
8. Gasser CS, Broadhvest J, Hauser BA: Genetic Analysis of Ovule Development. Annu Rev Plant Physiol Plant Mol Biol 1998, 49: I-24.

9. Broadhvest J, Baker SC, Gasser CS: SHORT INTEGUMENTS 2 promotes growth during Arabidopsis reproductive development. Genetics 2000, I 55(2):899-907.

10. Golden TA, Schauer SE, Lang JD, Pien S, Mushegian AR, Grossniklaus $U$, Meinke DW, Ray A: SHORT INTEGUMENTSII SUSPENSORI/CARPEL FACTORY, a Dicer homolog, is a maternal effect gene required for embryo development in Arabidopsis. Plant Physiol 2002, I 30(2):808-822.

II. Ray A, Lang JD, Golden T, Ray S: SHORT INTEGUMENT (SINI), a gene required for ovule development in Arabidopsis, also controls flowering time. Development 1996, I 22(9):263I-2638.

12. Chuck G, Lincoln C, Hake S: KNATI induces lobed leaves with ectopic meristems when overexpressed in Arabidopsis. Plant Cell 1996, 8(8): I 277-1289.

13. Lincoln C, Long J, Yamaguchi J, Serikawa K, Hake S: A knotted I-like homeobox gene in Arabidopsis is expressed in the vegetative meristem and dramatically alters leaf morphology when overexpressed in transgenic plants. Plant Cell 1994 6(12):1859-1876.

14. Haseloff J: GFP variants for multispectral imaging of living cells. Methods Cell Biol 1999, 58:139-151.

15. Skinner DJ, Hill TA, Gasser CS: Regulation of ovule development. Plant Cell 2004, 16 Suppl:S32-45.

16. Balasubramanian S, Schneitz K: NOZZLE regulates proximal-distal pattern formation, cell proliferation and early sporogenesis during ovule development in Arabidopsis thaliana. Development 2000, I 27(19):4227-4238.

17. Meister RJ, Kotow LM, Gasser CS: SUPERMAN attenuates positive INNER NO OUTER autoregulation to maintain polar development of Arabidopsis ovule outer integuments. Development 2002, I 29(1 8):428|-4289.

18. Sinha N, Hake S, Freeling M: Genetic and molecular analysis of leaf development. Curr Top Dev Biol 1993, 28:47-80.

19. Odell JT, Nagy F, Chua NH: Identification of DNA sequences required for activity of the cauliflower mosaic virus $35 \mathrm{~S}$ promoter. Nature 1985, 313(6005):810-812.

20. Kalderon D, Roberts BL, Richardson WD, Smith AE: A short amino acid sequence able to specify nuclear location. Cell I984, 39(3 Pt 2):499-509.

21. Truernit E, Siemering KR, Hodge S, Grbic V, Haseloff J: A map of KNAT gene expression in the Arabidopsis root. Plant Mol Biol 2006, 60 (I): $1-20$.

22. Cutler SR, Ehrhardt DW, Griffitts JS, Somerville CR: Random GFP::cDNA fusions enable visualization of subcellular structures in cells of Arabidopsis at a high frequency. Proc Natl Acad Sci U S A 2000, 97(7):3718-3723.

23. Kurup S, Runions J, Kohler U, Laplaze L, Hodge S, Haseloff J: Marking cell lineages in living tissues. Plant $J$ 2005, 42(3):444-453.

24. Bowman JL: Arabidopsis : an Atlas of Morphology and Development. Berlin \& New York, Springer-Verlag; 1993.

25. Byrne ME: Networks in leaf development. Curr Opin Plant Biol 2005, 8(I):59-66.

26. McConnell JR, Barton MK: Leaf polarity and meristem formation in Arabidopsis. Development 1998, I 25(I5):2935-2942

27. McConnell JR, Emery J, Eshed Y, Bao N, Bowman J, Barton MK: Role of PHABULOSA and PHAVOLUTA in determining radial patterning in shoots. Nature 200 I, 4 I I (6838):709-7I3.

28. Sieber P, Gheyselinck J, Gross-Hardt R, Laux T, Grossniklaus U, Schneitz K: Pattern formation during early ovule development in Arabidopsis thaliana. Dev Biol 2004, 273(2):32I-334.

29. Douglas SJ, Chuck G, Dengler RE, Pelecanda L, Riggs CD: KNATI and ERECTA regulate inflorescence architecture in Arabidopsis. Plant Cell 2002, I4(3):547-558.

30. Venglat SP, Dumonceaux T, Rozwadowski K, Parnell L, Babic V, Keller W, Martienssen R, Selvaraj G, Datla R: The homeobox gene BREVIPEDICELLUS is a key regulator of inflorescence architecture in Arabidopsis. Proc Natl Acad Sci U S A 2002, 99(7):4730-4735.

31. Meister RJ, Oldenhof H, Bowman JL, Gasser CS: Multiple protein regions contribute to differential activities of YABBY proteins in reproductive development. Plant Physiol 2005, 137(2):651-662.
32. Byrne ME, Simorowski J, Martienssen RA: ASYMMETRIC LEAVESI reveals knox gene redundancy in Arabidopsis. Development 2002, I29(8): 1957-1965.

33. Frugis G, Giannino D, Mele G, Nicolodi C, Chiappetta A, Bitonti MB Innocenti AM, Dewitte W, Van Onckelen H, Mariotti D: Overexpression of KNATI in lettuce shifts leaf determinate growth to a shoot-like indeterminate growth associated with an accumulation of isopentenyl-type cytokinins. Plant Physiol 200I, I 26(4): I370-1380.

34. Tsukaya $\mathrm{H}$ : Organ shape and size: a lesson from studies of leaf morphogenesis. Curr Opin Plant Biol 2003, 6(I):57-62.

35. Tsukaya $\mathrm{H}$ : Leaf shape: genetic controls and environmental factors. Int J Dev Biol 2005, 49(5-6):547-555.

36. Horiguchi G, Ferjani A, Fujikura U, Tsukaya $\mathrm{H}$ : Coordination of cell proliferation and cell expansion in the control of leaf size in Arabidopsis thaliana. J Plant Res 2006, I I 9(1):37-42.

37. Garcia D, Fitz Gerald JN, Berger F: Maternal Control of Integument Cell Elongation and Zygotic Control of Endosperm Growth Are Coordinated to Determine Seed Size in Arabidopsis. Plant Cell 2005, I7(I):52-60.

38. Website: [http://nasc.nott.ac.uk]

39. Koncz $C$ Schell, J.: The promoter of the TL-DNA gene 5 controls the tissue-specific expression of chimaeric genes carried by a novel type of Agrobacterium binary vector. Mol Gen Genet 1986, 204:383 -3396.

40. Clough SJ, Bent AF: Floral dip: a simplified method for Agrobacterium-mediated transformation of Arabidopsis thaliana. Plant J 1998, 16(6):735-743.

4I. Nesi N, Jond C, Debeaujon I, Caboche M, Lepiniec L: The Arabidopsis TT2 gene encodes an R2R3 MYB domain protein that acts as a key determinant for proanthocyanidin accumulation in developing seed. Plant Cell 200I, I3(9):2099-2I I4.

42. Website: [http://rsb.info.nih.gov/nih-image]

Publish with BioMed Central and every scientist can read your work free of charge

"BioMed Central will be the most significant development for disseminating the results of biomedical research in our lifetime. "

Sir Paul Nurse, Cancer Research UK

Your research papers will be:

- available free of charge to the entire biomedical community

- peer reviewed and published immediately upon acceptance

- cited in PubMed and archived on PubMed Central

- yours - you keep the copyright
BioMedcentral 